\title{
Luz, giberelinas y profundidad de siembra inciden sobre la germinación de semillas de Amaranthus hybridus $L$.
}

\section{Light, gibberellins and burial depth affect seed germination of Amaranthus hybridus L.}

\author{
Juan Camilo Niño-Hernandez'; David Felipe Moreno²; Hernán David Ruiz-Berrío ${ }^{3}$; \\ Helber Enrique Balaguera-López ${ }^{4}$; Stanislav Magnitskiy ${ }^{5}$
}

\begin{abstract}
11ng. Agrónomo. Universidad Pedagógica y Tecnológica de Colombia, Facultad de Ciencias Agropecuarias, Grupo de Investigaciones Agrícolas. Tunja -
\end{abstract} Boyacá, Colombia; e-mail: juan.nino03@uptc.edu.co; (D) https://orcid.org/0000-0002-8535-5331

${ }^{2}$ Ing. Agrónomo. Universidad Pedagógica y Tecnológica de Colombia, Facultad de Ciencias Agropecuarias. Tunja - Boyacá, Colombia; e-mail: david. moreno01@uptc.edu.co; q.e.p.d.

${ }^{3}$ Ing. Agrónomo. Universidad Pedagógica y Tecnológica de Colombia, Grupo de Investigaciones Agrícolas, Facultad de Ciencias Agropecuarias. Tunja Boyacá, Colombia; e-mail: hernan.ruiz@uptc.edu.co; (D) https://orcid.org/0000-0002-1450-7342

${ }^{4}$ Ing. Agrónomo, M.Sc., Ph.D. Universidad Nacional de Colombia, Facultad de Ciencias Agrarias, Grupo de Investigación Horticultura. Bogotá, Colombia; e-mail: hebalagueral@unal.edu.co; (D) https://orcid.org/0000-0003-3133-0355

${ }^{5}$ Agrónomo, M.Sc., Ph.D. Universidad Nacional de Colombia, Facultad de Ciencias Agrarias, Grupo de Investigación Horticultura. Bogotá, Colombia; e-mail: svmagnitskiy@unal.edu.co; (D) https://orcid.org/0000-0002-3715-1932

Cómo citar: Niño-Hernandez, J.C.; Moreno, D.F.; Ruiz-Berrío, H.D.; Balaguera-López, H.E.; Magnitskiy, S. 2020. Luz, giberelinas y profundidad de siembra inciden sobre la germinación de semillas de Amaranthus hybridus L. Rev. U.D.C.A Act. \& Div. Cient. 23(2):e1545. http://doi.org/10.31910/rudca.v23.n2.2020.1545

Artículo de acceso abierto publicado por Revista U.D.C.A Actualidad \& Divulgación Científica, bajo una licencia Creative Commons CC BY-NC 4.0

Publicación oficial de la Universidad de Ciencias Aplicadas y Ambientales U.D.C.A, Institución de Educación Superior Acreditada de Alta Calidad por el Ministerio de Educación Nacional.

Recibido: Mayo 7 de 2020 Aceptado: Julio 28 de 2020 Editado por: Ingeborg Zenner de Polanía

\section{RESUMEN}

Amaranthus bybridus L., conocido como bledo, es una planta maleza en una gran variedad de cultivos en el mundo, que se caracteriza por producir alto número de semillas latentes. Para generar estrategias de manejo sostenibles en esta especie es necesario comprender mejor la fisiología de sus semillas. Por tanto, el objetivo fue evaluar el efecto de la luz, de las giberelinas y la profundidad de la siembra sobre la germinación de semillas de bledo. En un primer experimento, se evaluaron cuatro tratamientos de luz (rojo, azul, sin luz y luz natural «testigo»); en el segundo, se imbibieron semillas en diferentes concentraciones de ácido giberélico (0, 200, 400 y $800 \mathrm{mg} \mathrm{L}^{-1}$ de
GA3) y, en un último experimento, se evaluaron ocho profundidades de siembra $(10,20,30,40,50,60,70$ y $80 \mathrm{~mm})$, utilizando turba como sustrato. Se encontró mayor porcentaje de germinación en oscuridad, indicando que son semillas fotoblásticas negativas. Para el experimento de giberelinas, el mejor resultado se obtuvo con 400 y $800 \mathrm{mg} \mathrm{L}^{-1}$ de ácido giberélico. En el tercer experimento, se observó alto porcentaje de emergencia de plántulas en las primeras profundidades $(10 \mathrm{~mm}$ a $30 \mathrm{~mm})$; a mayor profundidad hubo menor emergencia. La respuesta diferencial a la luz, a la aplicación de las giberelinas y el alto porcentaje de semillas viables que no germinaron permite concluir que semillas de bledo poseen latencia fisiológica. 
Palabras clave: Tetrazolio; Latencia; Hormonas; Semillas fotoblásticas; Manejo de malezas.

\section{ABSTRACT}

Amaranthus bybridus L., known as bledo, is a weed in a wide variety of crops worldwide, characterized by producing high number of seeds with dormancy. To generate sustainable management strategies of this species, it is necessary to better understand the physiology of its seeds. Therefore, the objective was to evaluate the effect of light, gibberellins, and seed burying depth on seed germination of bledo. In the first experiment, four light conditions were evaluated (red, blue, without light and natural light (control)), in the second experiment the seeds were imbibed in different concentrations of gibberellic acid (0, 200, 400 and $800 \mathrm{mg} \mathrm{L}^{-1}$ of $\left.\mathrm{GA}_{3}\right)$, and in the last experiment, 8 planting depths were evaluated (10, 20, 30, 40, 50, 60,70 or $80 \mathrm{~mm}$ ) using peat as a substrate. A higher percentage of seed germination in darkness was found, indicating that this species had negative photoblastic seeds. For the gibberellin experiment, the best result was obtained with treatment with 400 and $800 \mathrm{mg} \mathrm{L}^{-1}$ of gibberellic acid. In the third experiment, a high percentage of seedling emergence was observed from the first depths $(10 \mathrm{~mm}$ to $30 \mathrm{~mm})$; the greater was the depth, the less emergence was found. The differential responses to light, the application of gibberellins, and the high percentage of non-germinated viable seeds allowed to conclude that bledo seeds possess physiological dormancy.

Keywords: Tetrazolium; Dormancy; Hormones; Photoblastic seeds; Weed management.

\section{INTRODUCCIÓN}

El amaranto o bledo (Amaranthus bybridus L.) es una planta herbácea anual, ampliamente distribuida en Norteamérica, Europa y países tropicales. El género Amaranthus contiene más de 70 especies, de las cuales, la mayoría son nativas de América (Ruíz et al. 2018). El bledo es una de las malezas más limitantes, a nivel mundial, en varios cultivos (Zimdahl, 2018). Además, las especies de Amaranthus, se consideran como un posible cultivo futuro, porque son fuente importante de compuestos bioactivos, nutrientes y vitaminas. Las hojas de $A$. bybridus y $A$. caudatus, se utilizan como fuentes de alimento y en medicina tradicional, en países africanos e India (Sivakumar et al. 2018; Jimoh et al. 2019). Las plantas pertenecientes a estas especies, se adaptan rápidamente a condiciones ambientales adversas, como déficit hídrico y altas temperaturas y son fáciles de cultivar (Rastogi \& Shukla, 2013; Zhang et al. 2019). Actualmente, existe poca información de la fisiología y la ecología de Amaranthus, especialmente, de $A$. bybridus, como por ejemplo, de los factores que determinan la germinación de sus semillas. Esta información puede ser valiosa para generar estrategias de manejo integral de malezas, cuando $A$. bybridus está presente en los cultivos.

Varias especies pertenecientes al género Amaranthus son exitosas como malezas, porque presentan metabolismo fotosintético $\mathrm{C} 4 \mathrm{y}$ altas tasas de crecimiento. Producen grandes cantidades de semilla en sistemas agrícolas (entre 37.000 y 600.000 semillas por planta), muchas de ellas presentan latencia y poseen alta habilidad para la dispersión de semillas por el viento (Assad et al. 2017; Xavier et al. 2019). Esta condición permite que permanezcan viables en bancos de semillas en el suelo, por un largo periodo de tiempo (Assad et al. 2017; Xavier et al. 2019).

Estudios previos indicaron que la luz, la salinidad y la profundidad en el perfil del suelo pueden afectar la germinación de semillas de Amaranthus (Taylorson, 1991; Jimoh et al. 2019; Idris et al. 2020). Assad et al. (2017) observaron que la germinación fue mayor en los primeros $20 \mathrm{~mm}$ de profundidad del suelo. También, se ha reportado que las semillas recién dispersadas de la planta presentan alto vigor (Valdez-Eleuterio et al. 2015). Al mismo tiempo, muchos aspectos de la germinación de semillas de Amaranthus se desconocen. Esto toma mayor relevancia en función del conocimiento de que variables abióticas, tales como temperatura, condición lumínica y el contenido de agua del suelo, pueden promover, de manera diferencial, la respuesta fotoblástica entre poblaciones de Amaranthus (Castillo et al. 2013).

El requerimiento de luz, al igual que la dependencia de señales químicas del ambiente (nitrato, etileno, karrikinas u otros), son algunos de los factores que han sido citados como importantes, para desencadenar la germinación de semillas pequeñas, que posean muy pocas reservas de almacenamiento (Baskin \& Baskin, 2014). En el caso de que germinen demasiado profundo en el suelo, sus reservas nutricionales no alcanzan para sostener el crecimiento de las plántulas y que ésta llegue exitosamente a emerger a la superficie del suelo (Fenner, 2012).

También, otros factores distintos de la luz, han sido citados como reguladores de la germinación de las semillas (Savaedi et al. 2019), tales como las hormonas giberelinas, que controlan la germinación de semillas, a través de la activación de enzimas hidrolíticas y reacciones mediadas por fitocromos PhyA y PhyB (Barros-Galvão et al. 2019; Savaedi et al. 2019).

Por lo anterior, el objetivo fue evaluar la influencia de la luz, de la aplicación de giberelinas y la profundidad de siembra, sobre la capacidad de germinación y de viabilidad de las semillas del bledo Amaranthus bybridus L., en condiciones de laboratorio e invernadero.

\section{MATERIALES Y MÉTODOS}

Material vegetal y ubicación. En octubre de 2017, se recolectaron, aproximadamente, 5.500 semillas maduras de $A$. bybridus, en lotes agrícolas en barbecho, de Tunja y de Paipa (Boyacá, Colombia); cada semilla está contenida en un fruto seco, tipo utrículo. Los experimentos, se llevaron a cabo en el Laboratorio de Fisiología Vegetal y del invernadero de vidrio de la Facultad de Ciencias Agropecuarias de la Universidad Pedagógica y Tecnológica de Colombia, sede Tunja, que se encuentra a $2.782 \mathrm{~m}$ s.n.m., a $73^{\circ} 23^{\prime} \mathrm{W}$ y $5^{\circ} 32^{\prime} \mathrm{N}$.

Las semillas cosechadas, se dejaron secar al ambiente $\left(16 \pm 1,6^{\circ} \mathrm{C}\right)$ y se almacenaron a temperatura promedio de $18 \pm 1,9^{\circ} \mathrm{C}$, bajo condiciones de oscuridad, en bolsas de papel, durante 21 días. La 
muestra de semillas fue utilizada para realizar tres experimentos, cada uno, bajo un diseño completamente al azar, como se describe a continuación.

Experimento 1. Para determinar el efecto de la luz sobre la germinación, se evaluaron cuatro tratamientos, de acuerdo con Porras et al. (2020): luz roja, luz azul, oscuridad (sin luz) y con luz solar natural, como testigo. Cada tratamiento tuvo cuatro repeticiones y cada una estuvo compuesta de 100 semillas. Para los tratamientos de luz roja (640-670nm) y azul (450-510nm), se cubrieron las cajas Petri con papel celofán del respectivo color, mientras que para el tratamiento sin luz, se utilizó papel aluminio. Antes de la siembra en cada caja Petri, se dispuso filtro humedecido y se agregaron alrededor de $2 \mathrm{~mL}$ de agua destilada diariamente, a cada caja Petri. Se contó con luz natural $\left(211 \mu \mathrm{mol} \mathrm{m}^{-2} \mathrm{~s}^{-1}\right)$, con fotoperiodo de $12 \mathrm{~h}$ y una temperatura promedio de $18^{\circ} \mathrm{C}$, temperatura favorable para germinación del bledo (Aufhammer et al. 1998).

Experimento 2. Las semillas fueron imbibidas en soluciones con diferentes concentraciones de giberelinas $\left(0 \mathrm{mg} \mathrm{L}^{-1}, 200 \mathrm{mg} \mathrm{L}^{-1}\right.$, $400 \mathrm{mg} \mathrm{L}^{-1}, 600 \mathrm{mg} \mathrm{L}^{-1}$, y $800 \mathrm{mg} \mathrm{L}^{-1}$ de $\mathrm{GA}_{3}$ ). Se utilizaron cuatro repeticiones por tratamiento y cada una de las 20 unidades experimentales estuvo conformada por una caja Petri, con 100 semillas. Se prepararon cada una de las soluciones de giberelinas en agua destilada y con el producto ProGibb® 10 SP (Bayer S.A, Colombia), cuyo ingrediente activo es ácido giberélico $(100 \mathrm{~g} / \mathrm{kg})$. Diariamente, se hizo aplicación con los tratamientos a las cajas Petri, para mantener el papel de filtro humedecido.

Experimento 3. Se diseñaron ocho tratamientos correspondientes a diferentes profundidades de siembra $(10,20,30,40,50,60,70$ y $80 \mathrm{~mm})$, con cuatro repeticiones por tratamiento. Cada repetición estuvo compuesta de 50 semillas sembradas en materas de $2 \mathrm{~L}$ y se utilizó turba rubia PRO-MIX, como sustrato. La siembra de cada semilla se realizó, a través de un tubo de $5 \mathrm{~mm}$ de diámetro, que se insertó en el sustrato a la profundidad requerida en cada tratamiento; luego de la siembra, las semillas se cubrieron con el mismo sustrato. El sustrato, se mantuvo a temperatura promedio de $18 \pm 1,9^{\circ} \mathrm{C}$, con humedad a capacidad de campo, mediante riego diario con agua destilada. Este experimento, se llevó a cabo en invernadero de vidrio.

Variables. En cada uno de los experimentos, se registró diariamente el número de semillas germinadas y el número de días desde la germinación de la primera semilla y hasta germinación constante. Una semilla, se consideró germinada cuando la radícula ya visible midiera, como mínimo, $2 \mathrm{~mm}$ de longitud. En el experimento de profundidades de siembra, se midió el porcentaje de emergencia de plántulas, entendida como la salida del hipocótilo sobre la superficie del sustrato.

Para cada experimento, se calcularon los siguientes parámetros de germinación: el porcentaje de germinación (PG), como el cociente entre las semillas germinadas y las semillas sembradas, multiplicado por 100. También, se calcularon la velocidad media de germinación (VMG) y el tiempo medio de germinación (TMG), de acuerdo con Carranza et al. (2016), utilizando las siguientes ecuaciones:

$$
\mathrm{VMG}=\sum\left(\frac{\mathrm{N}_{\mathrm{i}}}{\mathrm{T}_{\mathrm{i}}}\right)
$$

ecuación 1

Donde, $\mathrm{N}_{\mathrm{i}}=$ Número de semillas emergidas en el i-ésimo día. $\mathrm{T}_{\mathrm{i}}=$ Tiempo en días, para la emergencia en el i-ésimo día.

$$
\mathrm{TMG}=\frac{\mathrm{N} *\left(\mathrm{~A}_{1}+\mathrm{A}_{2}+\ldots+\mathrm{A}_{\mathrm{X}}\right)}{\left(\mathrm{A}_{1} * \mathrm{~T}_{1}+\mathrm{A}_{2} * \mathrm{~T}_{2}+\ldots+\mathrm{A}_{\mathrm{X}} * \mathrm{~T}_{\mathrm{X}}\right)}
$$

ecuación 2

Donde, $N=$ Número de semillas germinadas. $A_{1}, A_{2}, \ldots$ A $:$ Número de semillas germinadas en el día 1 , en el día 2 y en el día x, respectivamente; $T_{1}, T_{2}, \ldots, T_{x}=$ Número de días entre la siembra y el día 1 de germinación, entre el día 2 y entre el día x, respectivamente.

Se determinó la viabilidad de semillas (\%) en las semillas que no germinaron en los experimentos 1 y 2, siguiendo la técnica recomendada por Paraíso et al. (2019); cada semilla fue cortada longitudinalmente con un escalpelo y con el uso de un estereoscopio. Posteriormente, las semillas se sumergieron en una solución de cloruro de 2,3,5-trifenil tetrazolio al 1\%, en cajas Petri y se llevaron a la cámara, a una temperatura de $37^{\circ} \mathrm{C}$, por un período de $2 \mathrm{~h}$, en condiciones de oscuridad. Al cabo de este tiempo, se hizo la evaluación topográfica con estereoscopio, de acuerdo con las categorías presentadas en la figura $1 \mathrm{y}$ recomendadas por Paraíso et al. (2019).

Para el experimento 1, adicionalmente, se calculó el índice de fotoblastismo (IF), de acuerdo con la siguiente ecuación (Castillo et al. 2013):

$$
\mathrm{IF}=\left(\mathrm{GO}_{\mathrm{O}}-\mathrm{G}_{\mathrm{L}}\right) /\left(\mathrm{GO}_{\mathrm{O}}+\mathrm{GL}_{\mathrm{L}}\right)
$$

Donde, Go es el porcentaje de germinación en oscuridad y GL es el porcentaje de germinación en luz. Este índice varía de 1 (fotoblastismo negativo) a -1 (fotoblastismo positivo); $\mathrm{IF}=0$ indica que la germinación no es dependiente de la luz (Castillo et al. 2013).

Análisis estadístico. Inicialmente, se realizaron pruebas de normalidad de los errores de Shapiro-Wilk y de homogeneidad de varianzas de Levene; las variables que cumplieron con estos supuestos, se sometieron a análisis de varianza. En el caso de presentarse diferencias significativas $(\mathrm{p} \leq 0,05)$, se realizó una prueba de comparación de medias, utilizando la prueba de Tukey ( $p \leq 0,05)$. Las variables que no cumplieron los supuestos, se sometieron a una transformación, mediante la función arco seno. Los análisis, se realizaron con el programa estadístico SPSS V.19.

\section{RESULTADOS Y DISCUSIÓN}

Experimento 1. Las semillas puestas en oscuridad presentaron el mayor PG $(56,75 \% \pm 2,4)$ y difirió estadísticamente $(\mathrm{p} \leq 0,05)$ del tratamiento testigo (luz natural), que obtuvo el menor PG $(1,25 \% \pm$ 0,48 ) (Figura 2A). Los tratamientos de semillas germinadas con luz roja y azul no presentaron diferencias estadísticas con el tratamiento testigo (Figura 2A). La VMG registró igual tendencia que el PG (Figura 2B). No hubo diferencias significativas $(p>0,05)$ en VMG entre los tratamientos con luz y el testigo y estos difirieron $(\mathrm{p} \leq 0,05)$ 


\begin{tabular}{|c|c|c|c|}
\hline $\begin{array}{c}\text { Tipo de } \\
\text { viabilidad }\end{array}$ & Descripción & Fotografía & Esquema \\
\hline Viables & $\begin{array}{l}\text { Semillas con tinción total } \\
\text { y uniforme }\end{array}$ & & \\
\hline Viables & $\begin{array}{l}\text { Semillas con tinción en más del } \\
80 \% \text { de la radícula y el embrión }\end{array}$ & & \\
\hline Dudosas & $\begin{array}{l}\text { Semillas teñidas en más del } 50 \% \\
\text { de los cotiledones y tinción } \\
\text { rosada en el } 50 \% \text { de la radícula }\end{array}$ & & \\
\hline \multirow[t]{2}{*}{ No viables } & Semillas sin tinción & & \\
\hline & & & \\
\hline
\end{tabular}

Figura 1. Clasificación de semillas de Amaranthus bybridus L., sometidas a la prueba de tetrazolio, para identificación de patrones topológicos. Cs: cubierta seminal; E: embrión; R: radícula; C: cotiledones; En: endospermo; PA: perispermo.

del tratamiento con oscuridad. Las semillas imbibidas bajo luz natural presentaron significativamente $(\mathrm{p} \leq 0,05)$ menor TMG $(5,98 \pm$ 0,03 días), que el resto de los tratamientos. Los tratamientos con luz no presentaron diferencias estadísticas entre ellos, en términos de TMG (Figura 2C).

La mayor germinación obtenida en la oscuridad y el IF alto $(0,956 \pm$ $0,017)$ indican que las semillas de $A$. bybridus se comportaron como fotoblásticas negativas, es decir, que germinan mejor en la oscuridad (Bewley et al. 2013). Esta condición, también es la responsable de que las semillas germinadas en oscuridad presentaron mayor VMG. Por otro lado, las estrechas diferencias en TMG obtenidas entre tratamientos pueden ser explicadas, porque en los tratamientos con luz, las pocas semillas que germinaron lo hicieron en el mismo tiempo que las semillas germinadas en ausencia de luz. Para semillas de $A$. bybridus el fotoblastismo negativo puede representar una característica adaptativa, que previene la germinación de semillas sobre la superficie del suelo o en ausencia de la sombra de plantas, que pudieran ofrecer el microclima, adecuado para el desarrollo de la plántula, reduciendo su mortalidad (Castillo et al. 2013). Al respecto, en Eschscholzia californica Cham., especie invasora, que crece primariamente en sitios abiertos e intervenidos, también, se presenta semillas con fotoblastismo negativo (Castillo et al. 2013).

El fotoblastismo negativo puede ser modulado por el tipo de fitocromo presente en las semillas. Un caso es la presencia del fitocromo PhyA que controla la germinación, a través de respuestas de alta luminosidad (Takaki, 2001). En el caso de malezas, Kulkarni et al. (2006) sugieren que las semillas de Eucomi autumnalis son negativamente fotoblásticas, al igual que $A$. bybridus e indican que este tipo de fotoblastismo no es un fenómeno común en las semillas. A pesar de esta afirmación de Kulkarni et al. (2006), otros autores han reportado el fotoblastismo negativo en Amaranthus albus (Taylorson, 1991) y en $A$. caudatus L. (Kendrick \& Frankland, 1969) y concluyeron que el fitocromo controla la germinación de semillas fotoblásticas negativas, a través de respuestas de alta irradiación. 

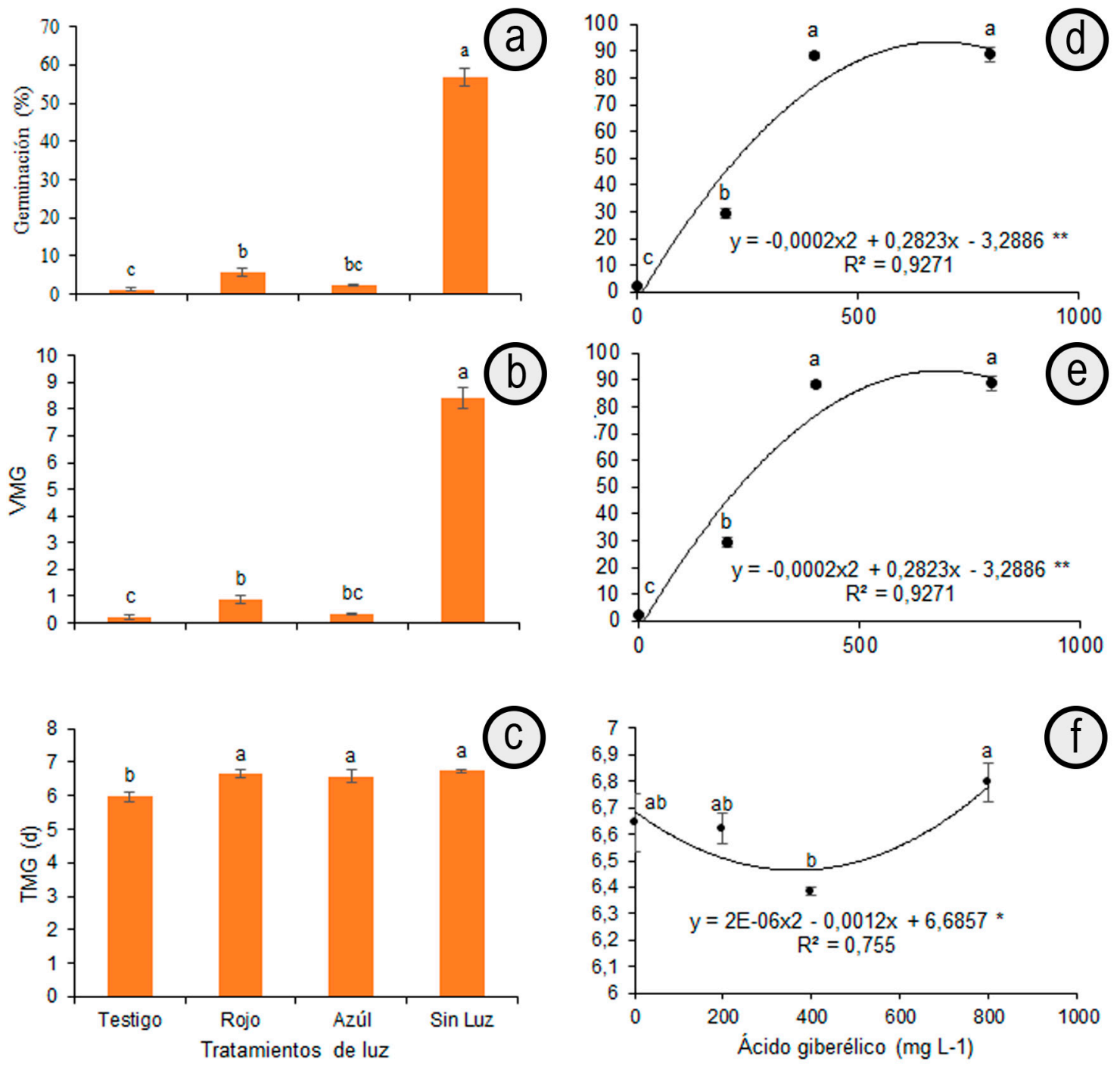

Figura 2. Efecto de diferentes tratamientos de luz sobre: a. porcentaje de germinación (PG); b. velocidad media de germinación (semillas germinadas/d) (VMG); c. tiempo medio de germinación (TMG). Efecto de la concentración ácido giberélico sobre: d. porcentaje de germinación; e. velocidad media de germinación (semillas germinadas/d); f. tiempo medio de germinación de semillas de Amarantbus bybridus, en condiciones de laboratorio. Promedios con letras distintas indican diferencia significativa según la prueba de Tukey $(p \leq 0,05)$. Barras verticales en cada promedio indican el error estándar $(n=4)$.

En otras especies, la oscuridad es también favorable para la germinación (Penfield, 2017) y la germinación estaría bloqueada mediante la luz azul percibida por los fitocromos (Stawska \& Oracz, 2019) o los criptocromos (Penfield, 2017). Estos fotorreceptores podrían estar involucrados en acelerar la degradación de giberelinas en el embrión, manteniendo la semilla latente (Penfield, 2017). Esta situación podría explicar la inhibición de germinación obtenida en semillas de $A$. bybridus, con el tratamiento de luz azul (Figura 2A). También, se indica que las semillas con alta proporción del fitocromo en su conformación activa (Pfr), a menudo, pueden germinar en la oscuridad, porque ya han excedido el umbral requerido de Pfr para la germinación (Bewley et al. 2013).

Con relación a la viabilidad de las semillas no germinadas, se observó un alto porcentaje de semillas con alta viabilidad (viables
100\%) (Tabla 1). Las semillas bajo tratamiento con luz roja y luz azul, respectivamente presentaron mayor viabilidad; por el contrario, se observó que la menor viabilidad $(\mathrm{p}<0,01)$, se dio en semillas sometidas a oscuridad. Con relación a la tinción $80 \%$, las semillas germinadas bajo la luz ambiente presentaron significativamente la mayor viabilidad, mientras que con el tratamiento de luz roja o en oscuridad, se obtuvieron los menores valores. En semillas con viabilidad dudosa y las no viables, las semillas con luz ambiente tuvieron mayor porcentaje $(24,75 \pm 2,5 \%$ y $5,0 \pm 0,9 \%$, respectivamente); los demás tratamientos de luz fueron estadísticamente inferiores (Tabla 1). Las semillas no viables fue la categoría con menor porcentaje, indicando que las semillas recién cosechadas de $A$. bybridus posean alta viabilidad y que un alto porcentaje de las semillas que no germinan estarían en condiciones de latencia. Estos resultados concuerdan con los encontrados por Flores-Córdova et al. (2016), 
Tabla 1. Porcentajes de viabilidad mediante la prueba topográfica por tetrazolio, en semillas no germinadas de Amaranthus bybridus L., sometidas a diferentes tratamientos de luz y de ácido giberélico (GA3).

\begin{tabular}{|l|c|c|c|c|}
\hline \multirow{2}{*}{ Tratamiento de luz } & \multicolumn{4}{|c|}{ Categoría de viabilidad } \\
\cline { 2 - 5 } & Viable $\mathbf{1 0 0} \%$ & Viable $\mathbf{8 0} \%$ & Dudosa $\mathbf{5 0} \%$ & No viable \\
\hline Luz ambiente & $46,7 \pm 4,0 \mathrm{ab}$ & $22,2 \pm 2,3 \mathrm{a}$ & $24,8 \pm 2,5 \mathrm{a}$ & $5,0 \pm 0,9 \mathrm{a}$ \\
\hline Rojo & $67,5 \pm 3,4 \mathrm{a}$ & $13,0 \pm 0,9 \mathrm{~b}$ & $11,5 \pm 2,6 \mathrm{~b}$ & $2,2 \pm 0,6 \mathrm{~b}$ \\
\hline Azul & $70,5 \pm 2,9 \mathrm{a}$ & $15,2 \pm 2,6 \mathrm{ab}$ & $10,5 \pm 2,9 \mathrm{~b}$ & $1,5 \pm 0,3 \mathrm{~b}$ \\
\hline Sin luz & $27,7 \pm 3,0 \mathrm{~b}$ & $10,0 \pm 1,1 \mathrm{~b}$ & $3,7 \pm 0,7 \mathrm{~b}$ & $1,7 \pm 0,4 \mathrm{~b}$ \\
\hline Concentración de $\mathbf{G A}_{\left.\mathbf{~} \mathbf{(} \mathbf{m g ~ L}^{-1} \mathbf{)}\right)}$ & $42,0 \pm 4,7 \mathrm{a}$ & $33,5 \pm 6,5 \mathrm{a}$ & $18,5 \pm 2,2 \mathrm{a}$ & $3,5 \pm 0,6 \mathrm{a}$ \\
\hline 0 & $47,0 \pm 9,4 \mathrm{a}$ & $14,0 \pm 7,7 \mathrm{ab}$ & $8,5 \pm 1,9 \mathrm{~b}$ & $1,0 \pm 0,4 \mathrm{~b}$ \\
\hline 200 & $3,2 \pm 0,9 \mathrm{~b}$ & $2,7 \pm 0,7 \mathrm{~b}$ & $4,3 \pm 0,7 \mathrm{c}$ & $1,5 \pm 0,9 \mathrm{~b}$ \\
\hline 400 & $2,5 \pm 0,8 \mathrm{~b}$ & $3,0 \pm 1,1 \mathrm{~b}$ & $2,5 \pm 1,5 \mathrm{c}$ & $3,3 \pm 0,5 \mathrm{a}$ \\
\hline 800 &
\end{tabular}

Promedios con letras distintas en la misma columna para cada experimento presentan diferencias estadísticas, según la prueba de Tukey $(\mathrm{p} \leq 0,05)$.

quienes hallaron latencia similar en semilla forrajeras y de malezas, incluyendo a $A$. hybridus.

Experimento 2. Se observaron diferencias estadísticas significativas $(p<0,01)$ en el porcentaje de semillas germinadas a diferentes concentraciones de ácido giberélico. Este comportamiento, se ajustó a una función polinomial de segundo grado (Figura 2D). Las semillas que no fueron tratadas con giberelinas presentaron menor porcentaje de germinación $(2,5 \pm 0,28 \%)$. Los valores de germinación más altos, se presentaron en las semillas tratadas con 800 y $400 \mathrm{mg} \mathrm{L}^{-1}$ de ácido giberélico (88,75 $\pm 3,56 \%$ y 88,25 $\pm 1,31 \%$, respectivamente) (Figura 2D).

La VMG en función del tratamiento con giberelinas, se explicó por una función cuadrática. Aquí, también, se observaron diferencias estadísticas significativas $(p<0,01)$ entre tratamientos. Las semillas sin aplicación de giberelinas (testigo) presentaron la menor VMG $(0,37 \pm 0,04$ semillas germinadas/d), en comparación con las semillas expuestas a concentraciones de 400 y $800 \mathrm{mg} \mathrm{L}^{-1}$ de ácido giberélico $(13,82 \pm 0,23$ y $13,08 \pm 0,65$ semillas germinadas/d, respectivamente) (Figura 2E). El TMG disminuyó cuando aumentó la concentración de ácido giberélico entre 0 a 400 $\mathrm{mg} \mathrm{L}^{-1}$, pero luego hubo un incremento significativo y alcanzó su máximo valor $(6,79 \pm 0,07 \mathrm{~d})$, al utilizar $800 \mathrm{mg} \mathrm{L}^{-1}$. Este comportamiento fue descrito por una función polinomial de segundo grado (Figura 2F).

Estos resultados sugieren que $A$. bybridus presenta latencia fisiológica (Baskin \& Baskin, 2004), condición que, en muchas especies, está regulada por altos niveles de ácido abscísico $(\mathrm{ABA})$ y bajos de giberelinas en el embrión (Penfield, 2017). Esta condición de latencia implica la activación de dos proteínas represoras de la germinación, como RGA-LIKE2 (RGL2) y DELLA, pero cuando las giberelinas se unen a su receptor GA-INSENSITIVE DWARF (GID1), se desencadena una serie de señales, que conllevan a la degradación o inactivación de RGL2 y esto, a su vez, libera a la semilla de la laten- cia e induce la germinación (Bewley et al. 2013). A su vez, los altos niveles de giberelinas en los tratamientos, también promueven la germinación de semillas, al estimular la síntesis de enzimas hidrolíticas, que degradan las sustancias de reserva y debilitan la integridad de cubiertas seminales, favoreciendo la salida de radícula, a través de aquellos durante la germinación (Shu et al. 2016). Estos procesos no solo generan mayor germinación, también logran que ocurra a mayor velocidad y en menor tiempo, como posiblemente ocurrió en las semillas de $A$. bibridus. En concordancia con los resultados aquí presentados, Martínez et al. (2016) encontraron altos porcentajes de germinación en semillas de Annona squamosa, tratadas con $800 \mathrm{mg}$ $\mathrm{L}^{-1}$ de GA3. Yang et al. (2020), también reportan que la aplicación de GA3 fue efectiva para inducir la germinación Primula beesiana.

Las categorías de semillas viables (100 y 80\% de tinción) fueron las más representativas, seguida de semillas dudosas, mientras que las semillas no viables presentaron un bajo porcentaje. El hecho de que las semillas de $A$. bybridus se mantuvieron viables, pero no germinaron en las condiciones consideradas favorables $\left(18^{\circ} \mathrm{C}\right)$ para esta especie (Aufhammer et al. 1998), ratifica que estas semillas presentaron latencia. Las semillas sin adición de giberelinas presentaron mayores porcentajes de viabilidad, para las categorías 100\% tinción y $80 \%$ tinción, respectivamente, pero, también, presentaron los mayores valores en las categorías dudosas y no viables. Por el contrario, con 400 y $800 \mathrm{mg} \mathrm{L}^{-1}$ del GA3, se encontraron los porcentajes más bajos $(p<0,01)$ de semillas viables y dudosas y con $800 \mathrm{mg} \mathrm{L}^{-1}$, la cantidad de semillas no viables fue similar al testigo (Tabla 1 ), pero los porcentajes en estos tratamientos hormonales fueron muy bajos, porque la mayoría de las semillas germinaron (Figura 2).

Los resultados de los experimentos 1 y 2 ponen en evidencia que la latencia de semillas puede ser liberada por factores fisiológicos (p.e. $\mathrm{GA}_{3}$ ) y ambientales (condiciones de luz), tal como lo plantearon 
Baskin \& Baskin (2004) y Yang et al. (2020). También es para destacar que la germinación de semillas de $A$. hybridus, con adición de giberelinas, en el experimento 2, fue mayor que la germinación de semillas en el experimento 1, en oscuridad o tratadas con diferentes tipos de luz. Este resultado es indicativo del indispensable rol de las giberelinas en la germinación de semillas de $A$. bybridus, no solo por la activación de enzimas hidrolíticas, sino, también, porque están involucradas en las funciones de los fotorreceptores (Savaedi et al. 2019), teniendo en cuenta que la síntesis de giberelinas en semillas fotoblásticas es controlada por los fotorreceptores (Stawska \& Oracz, 2019).En este sentido, distintos autores sugieren que las giberelinas pueden actuar como una alternativa parcial para reemplazar el requerimiento de luz (Bewley et al. 2013) o de oscuridad en A. hybridus (Mérai et al. 2019). Esta información cobra relevancia, puesto que la adición de giberelinas se convierte en una herramienta alternativa, que permita liberar a las semillas de $A$. bybridus de su latencia (Tabla 1) y ayude a entender los requerimientos fisiológicos de germinación de esta especie, con fines ecológicos o de manejo en sistemas agrícolas.

Experimento 3. Los resultados de emergencia de semillas de $A$. bybridus en relación con la profundidad de siembra, se ajustó a una función polinomial de tercer grado. El porcentaje de emergencia fue mayor $(p<0,01)$ en semillas, que estuvieron a 10 y a $30 \mathrm{~mm}$ de profundidad, con valores cercanos al $50 \%$. A profundidades mayores a los $30 \mathrm{~mm}$, la germinación se disminuyó gradualmente, hasta hacerse nula, a $80 \mathrm{~mm}$ de profundidad (Figura 3A). La velocidad media de emergencia presentó diferencias significativas $(p<0,05)$, entre profundidad de siembra y fue mayor entre los 10 y $30 \mathrm{~mm}$ de profundidad. Entre los 40 y $70 \mathrm{~mm}$ de profundidad, la velocidad disminuyó, hasta ser cero, a $80 \mathrm{~mm}$ de profundidad, dado que no hubo emergencia de plántulas. Este comportamiento, se ajustó a una ecuación polinomial de tercer grado (Figura 3B). Los mayores tiempos medios de emergencia, se obtuvieron en las profundidades
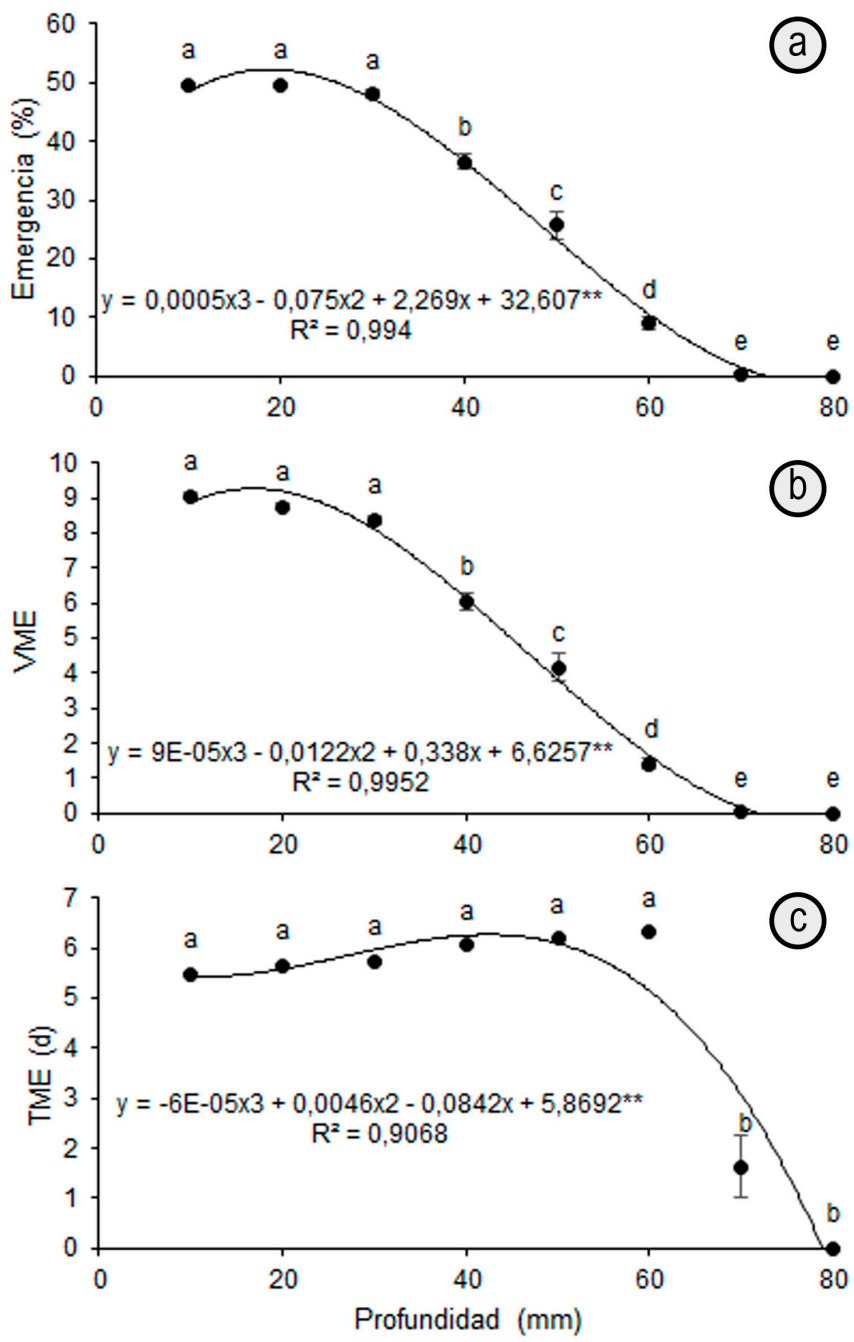

Figura 3. Efecto de la profundidad de siembra sobre: a. porcentaje de emergencia; b. velocidad media de emergencia (VME) (plántulas emergidas/d); c. tiempo medio de emergencia (TME) de semillas de $A$. bybridus, en condiciones de laboratorio. Medias con letras distintas indican diferencia significativa según la prueba de Tukey $(\mathrm{p} \leq 0,05)$. Barras verticales en cada promedio indican el error estándar $(\mathrm{n}=4)$. ** regresión significativa $\mathrm{p} \leq 0,01$. 
de $10 \mathrm{a} 60 \mathrm{~mm}$. A profundidades superiores, los tiempos fueron significativamente $(p<0,01)$ inferiores (Figura 3C).

Varios estudios previos coinciden con los resultados aquí reportados. Valdez-Eleuterio et al. (2015) reportaron que $A$. bybridus tiende a tener una mayor capacidad de germinación cuando se encuentra a menor profundidad del suelo $(10 \mathrm{~mm})$. En semillas de $A$. caudatus, Jimoh et al. (2019) encontraron que la mejor germinación se produjo en profundidades ente 10 y $20 \mathrm{~mm}$. Por su parte, Li et al. (2020) reportaron que semillas de Peripoca sepium germinaron a mayor velocidad, cuando estuvieron a $20 \mathrm{~mm}$ de profundidad, mientras que a profundidades de 40-50 mm, la velocidad disminuyó considerablemente.

De 10 a $30 \mathrm{~mm}$ de profundidad hay condiciones favorables para la germinación de $A$. bybridus, esto implica presencia de humedad, oxígeno y oscuridad (Baskin \& Baskin, 2014), a profundidades mayores; a pesar de continuar la condición de oscuridad y de humedad, deben existir otros factores que estarían siendo más limitantes para la emergencia de las plántulas. Estos factores pueden incluir la concentración del oxígeno y la misma presencia de luz, pues aunque la oscuridad favoreció a la germinación, después de la salida del hipocótilo es necesaria la presencia de luz para continuar con el proceso de fotomorgénesis y evitar la etiolación.

Fue evidente que las plántulas que emergieron a mayor profundidad presentaron tallos más alargados (datos no mostrados), como respuesta a la falta de luz. Al respecto, Bewley et al. (2013) mencionan que los requisitos de luz de las semillas se modifican fuertemente por otros factores ambientales, como la temperatura y otros factores del suelo, como lo es el contenido de nitrato en solución, que afectarán el comportamiento de las semillas en el campo. Además, es importante que estas semillas germinen cerca de la superficie, para que sus reservas limitadas puedan sostener el desarrollo de plántulas, antes de que estas comienzan a fotosintetizar (Bewley et al. 2013).

Con el aumento de la profundidad, las semillas presentes en el banco de semillas del suelo son expuestas a más bajos niveles de oxígeno, más altas concentraciones de $\mathrm{CO}_{2}$, ausencia de luz, temperaturas más bajas y estables. Bajo estas condiciones, las semillas pueden permanecer quiescentes o adquirir latencia secundaria (Baskin \& Baskin, 2004). Ambas condiciones son favorables para prolongar la permanencia de las semillas en un banco y, en particular, para formar un banco persistente de semillas (Baskin \& Baskin, 2004). En campo sería probable encontrar semillas de $A$. bybridus bajo estas condiciones; esto permitiría, que las semillas de $A$. bybridus pueden estar viables por varios años y que, luego por efecto de alguna labranza del suelo, se pueden quedar cerca de la superficie y activar la germinación. La dinámica de mortalidad y de supervivencia de semillas de $A$. hybridus en los bancos de semillas de suelos agrícolas es poco conocida, pero merece ser estudiada a futuro, debido a sus implicancias prácticas en el manejo de malezas de sistemas de producción de cultivos.

Los resultados de esta investigación permiten concluir que las semillas de bledo son semillas fotoblásticas negativas. La respuesta diferencial a la luz y aplicación de giberelinas exógenas permiten afirmar que estas semillas presentan latencia fisiológica. La alta proporción de semillas no germinadas que estaban viables refuerzan estos resultados. Se observó buena emergencia de plántulas a profundidades de 10 a $30 \mathrm{~mm}$ del sustrato, mientras que, a mayor profundidad, la germinación se vio comprometida.

Agradecimientos: Este manuscrito fue parte del trabajo de grado del Ing. Agr., David Felipe Moreno Benítez (q.e.p.d.), quién en vida contribuyó, de manera importante, al desarrollo del agro en Colombia. David, paz en tu tumba, permanecerás por siempre en nuestra memoria. Conflicto de intereses: El manuscrito fue preparado y revisado con la participación de todos los autores, quienes declaramos que no existe conflicto de intereses que ponga en riesgo la validez de los resultados presentados.

\section{REFERENCIAS}

1. ASSAD, R.; RESHI, Z.A.; JAN, S.; RASHID, I. 2017. Biology of Amaranths. The Botanical Review. 83(4):382-436. https://doi.org/10.1007/s12229-017-9194-1

2. AUFHAMMER, W.; CZUCZOROVA, D.; KAUL, H.-P.; KRUSE, M. 1998. Germination of grain amaranth (Amaranthus bypochondriacus $\times A$. bybridus): effects of seed quality, temperature, light, and pesticides. European J. Agronomy, 8(1-2):127-135. https://doi.org/10.1016/s1161-0301(97)00049-x

3. BARROS-GALVÃO, T.; DAVE, A.; GILDAY, A.D.; HARVEY, D.; VAISTIJ, F.E.; GRAHAM, I.A. 2019. ABA INSENSITIVE4 promotes rather than represses PHYA-dependent seed germination in Arabidopsis thaliana. New Phytologist. 226:953-956.

https://doi.org/10.1111/nph.16363

4. BASKIN, C.C.; BASKIN, J.M. 2014. Seeds: Ecology, Biogeography, and Evolution of Dormancy and Germination. Second ed. Academic Press (San Diego). 169p.

5. BASKIN, J.M.; BASKIN, C.C. 2004. A classification system for seed dormancy. Seed Science Research. (U.K). 14(1):1-16. https://doi.org/10.1079/SSR2003150

6. BEWLEY, D.J.; BRADFORD, K.J.; HILHORST, H.W.M.; NONOGAKI, H. 2013. Seeds: Physiology of Development, Germination and Dormancy. 3rd ed. Springer Science. 399p.

7. CARRANZA, C.; CASTELLANOS, G.; DEAZA, D.; MIRANDA, D. 2016. Efecto de la aplicación de reguladores de crecimiento sobre la germinación de semillas de badea (Passiflora quadrangularis L.) en condiciones de invernadero. Revista Colombiana de Ciencias Hortícolas, 10(2): 284-291. https://doi.org/10.17584/rcch.2016v10i2.5791 
8. CASTILlO, M.; BUSTAMANTE, O.R.; PEÑA-GÓMEZ, F.T.; GUTIÉRREZ, V.L.; REYES, C.A.; ARREDONDO-NÚÑEZ, A.; MAREY, M. 2013. Negative photoblastism in the invasive species Eschscholzia californica Cham. (Papaveraceae): Patterns of altitudinal variation in native and invasive range. Gayana Botanica. 70(2):330-336. https:/ / doi.org/10.4067/S0717-66432013000200010

9. FENNER, M.W. 2012. Seed Ecology. Springer Science \& Business Media. 151p.

10. FLORES-CÓRDOVA, M.A.; SÁNCHEZ-CHÁVEZ, E.; BALANDRÁN-VALLADARES, M.I.; MÁRQUEZ-QUIROZ, C. 2016. Efectividad de tratamientos pre-germinativos en la ruptura de la dormancia en las semillas forrajeras y de malezas. Ecosistemas y recursos agropecuarios. 3(9):427432.

11. IDRIS, L.M.; NULIT, R.; ZAMAN, F.Q.; ARIFIN, F.K.M. 2020. Hydrotime analysis of Amaranthus spp. seed germination under salinity condition. Journal of Applied Research on Medicinal and Aromatic Plants. In Press. https://doi.org/10.1016/j.jarmap.2020.100249

12. JIMOH, M.O.; AFOLAYAN, A.J.; LEWU, F.B. 2019. Germination response of Amaranthus caudatus L. to soil types and environmental conditions. Thaiszia J. Botany. 29(1):85-100. https://doi.org/10.33542/TJB2019-1-07

13. KENDRICK, R.E.; FRANKLAND, B. 1969. Phytocontrol of germination in Amaranthus caudatus. Planta. 85:326329.

14. KULKARNI, M.G.; SPARG, S.G.; VAN STADEN. J. 2006. Dark conditioning, cold stratification and a smoke-derived compound enhance the germination of Eucomis autumnalis subsp. autumnalis seeds. South African J. Botany. 72:157-162. https://doi.org/10.1016/j.sajb.2005.06.006

15. LI, T.; SUN, J.; YANG, H.; LIU, J.; XIA, J.; SHAO, P. 2020. Effects of shell sand burial on seedling emergence, growth and stoichiometry of Periploca sepium Bunge. BMC Plant Biol. 20:112.

https://doi.org/10.1186/s12870-020-2319-4

16. MARTÍNEZ M., F.E.; MIRANDA L., D.; MAGNITSKIY, S. 2016. Sugar apple (Annona squamosa L.) seed germination affected by the application of gibberellins. Agronomía Colombiana. 34(1):17-24.

https://dx.doi.org/10.15446/agron.colomb.v34n1.53074

17. MÉRAI, Z.; GRAEBER, K.; WILHELMSSON, P.; ULLRICH, K.K.; ARSHAD, W.; GROSCHE, C.; TARKOWSKÁ, D.; TUREČKOVÁ, V.; STRNAD, M.; RENSING, S.A.; LEUBNER-METZGER, G.; SCHEID, O. 2019. Aethionema arabicum: a novel model plant to study the light control of seed germination. J. Experimental Botany, 70(12):3313-3328.

https://doi.org/10.1093/jxb/erz146

18. PARAÍSO, H.A.; BRANDÃO JR, D.D.S.; AVELAR, R.I.; C., COSTA, A. DA.; GOMES, L.S.; NASCIMENTO, W.M. 2019. Adjustments in the tetrazolium test methodology for assessing the physiological quality of chickpea seeds. J. Seed Science. 41(1):7-12. https://doi.org/10.1590/2317-1545v41n1187777

19. PENFIELD, S. 2017.Seed dormancy and germination. Current Biology. 27(17):874-878. https://doi.org/10.1016/j.cub.2017.05.050

20. PORRAS, Y.; PEDREROS, M.; REYES-ARDILA, W.; BALAGUERA-LOPEZ, H. 2020. Light Effect on germination of Champa (Campomanesia lineatifolia R. \& P.) seeds. Ciencia y Agricultura, 17(2):23-31. https://doi.org/10.19053/01228420.v17.n2.2020.10979

21. RASTOGI, A.; SHUKLA, S. 2013. Amaranth: a new millennium crop of nutraceutical values. Critical Review in Food Science and Nutrition. 53(2):109-125. https://doi.org/10.1080/10408398.2010.517876

22. RUÍZ HERNÁNDEZ, V.C.; LEGARÍA SOLANO, J.P.; SAHAGÚN CASTELLANOS, J.; DE LAO OLAN, M. 2018. Variabilidad genética en algunas especies cultivadas y silvestres de amaranto. Rev. Mex. Ciencias Agrícolas. $9(2): 405-416$. https://doi.org/10.29312/remexca.v9i2.1081

23. SAVAEDI, Z.; PARMOON, G.; MOOSAVI, S.A.; BAKHSHANDE, A. 2019. The role of light and Gibberellic Acid on cardinal temperatures and thermal time required for germination of Charnushka (Nigella sativa) seed. Industrial Crops \& Products. 132:140-149. https://doi.org/10.1016/j.indcrop.2019.02.025

24. SHU, K.; LIU, X.D.; XIE, Q.; HE, Z.H. 2016. Two faces of one seed: hormonal regulation of dormancy and germination. Molecular Plant. 9(1):34-45. https://doi.org/10.1016/j.molp.2015.08.010

25. SIVAKUMAR, D.; CHEN, L.; SULTANBAWA, Y. 2018. A comprehensive review on beneficial dietary phytochemicals in common traditional Southern African leafy vegetables. Food science \& nutrition. 6(4):714-727. https://doi.org/10.1002/fsn3.643

26. STAWSKA, M.; ORACZ, K. 2019. PhyB and HY5 are involved in the blue light-mediated alleviation of dormancy of Arabidopsis seeds possibly via the modulation of expression of 
genes related to light, GA, and ABA. Internal J. Molecular Sciences. 20(23):5882.

https://doi.org/10.3390/ijms20235882

27. TAKAKI, M. 2001. New proposal of classification of seeds based on forms of phytochrome instead of photoblastism. Rev. Brasileira de Fisiología Vegetal. 13(1):103-107. https://doi.org/10.1590/S0103-31312001000100011

28. TAYLORSON, R.B. 1991. Inhibition of germination in Amaranthus albus seed by prolonged irradiation: a physiological basis. Seed Science Research. 1(1):51-56.

https://doi.org/10.1017/S0960258500000635

29. VALDEZ-ELEUTERIO, G.; USCANGA-MORTERA, E.; KOHASHI-SHIBATA, J.; GARCÍA-NAVA, R.; MARTÍNEZ-MORENO, D.; TORRES-GARCÍA, J.; GARCÍA-ESTEVA, A. 2015. Tamaño de semilla, granulometría del sustrato y profundidad de siembra en el vigor de semilla y plántula de dos malezas. Agrociencia. 49(8):899-915.
30. XAVIER, J.B.; ANDRADE, D.B.D.; SOUZA, D.C.D.; GUIMARÃES, G.C.; RESENDE, L.V.; GUIMARÃES, R.M. 2019. Morphological, chemical and physiological characterization of Amaranthus spp. Seeds.J. Seed Science. 41(4):478487.

https://doi.org/10.1590/2317-1545v41n4226286

31. YANG, L.; PENG, D.; ZHI-MIN, L.; HUANG, L.; YANG, J.; SUN, H. 2020. Cold stratification, temperature, light, GA3, and $\mathrm{KNO}_{3}$ effects on seed germination of Primula beesiana from Yunnan, China. Plant Diversity. In press. https://doi.org/10.1016/j.pld.2020.01.003

32. ZHANG, Z.S.; KANG, Y.J.; CHE, L. 2019. Composition and thermal characteristics of seed oil obtained from Chinese amaranth. LWT-Food Science and Technology. 111:39-45. https://doi.org/10.1016/j.lwt.2019.05.007

33. ZIMDAHL, R.L. 2018. Fundamentals of weed science. 5th ed. Academic Press. 785p. 